\title{
Another Look at Skeletal Maturation Using Hand Wrist and Cervical Vertebrae Evaluation
}

\author{
Thomas J. Cangialosi ${ }^{1,2 *}$, V. Jason Vives ${ }^{3}$ \\ ${ }^{1}$ School of Dental Medicine, Department of Orthodontics, Rutgers University, Newark, New Jersey, USA \\ ${ }^{2}$ College of Dental Medicine, Division of Orthodontics, Columbia University, New York City, New York, USA \\ ${ }^{3}$ Private Practice of Orthodontics, Old Bridge, New Jersey, USA \\ Email: *cangiatj@sdm.rutgers.edu
}

How to cite this paper: Cangialosi, T.J. and Vives, V.J. (2018) Another Look at Skeletal Maturation Using Hand Wrist and Cervical Vertebrae Evaluation. Open Journal of Orthopedics, 8, 1-10. https://doi.org/10.4236/ojo.2018.81001

Received: November 7, 2017

Accepted: January 8, 2018

Published: January 11, 2018

Copyright $\odot 2018$ by authors and Scientific Research Publishing Inc. This work is licensed under the Creative Commons Attribution International License (CC BY 4.0).

http://creativecommons.org/licenses/by/4.0/

c) (i) Open Access

\begin{abstract}
Introduction and Aims-The Growth stage of a patient can have considerable influence on diagnosis, treatment goals, timing and planning and the eventual outcome of orthodontic/orthopedic treatment. The purpose of this study was to analyze associations between the cervical vertebrae maturation score (CVMS) and skeletal maturation index (SMI). The second objective was to determine the reproducibility of the measurements on lateral cephalograms and hand-wrist radiographs. Materials and Methods-Lateral cephalometric and left hand-wrist radiographs of 92 untreated subjects (44 females and 48 males) aged from 8 to 17 years were obtained from the files of the Columbia University, Division of Orthodontics and measured for growth stage using cervical vertebrae and hand-wrist methods. Results-A high correlation was revealed between the hand-wrist and cervical vertebrae measurements. The Spearman's rho correlation coefficient was 0.925 and significant at the 0.01 level. The correlation between hand-wrist and age $(0.665, \mathrm{p}<0.01)$ was slightly greater than that of the CVMS $(0.611, \mathrm{p}<0.01)$. Intra rater reliability was high. When the three categorically modified methods of the Fishman's 11 skeletal maturation stages in hand and wrist analysis were used to compare with CVMS, methods 2 and 3 were both statistically significantly different according to the Wilcoxon signed ranks test and the Sign test at a significance level of less than 0.05 . However, for method 1 , the tests showed probability scores of 0.028 and 0.151 , respectively, showing no significant difference at the 0.01 level in the Wilcoxon Signed Ranks Test, and no significant difference in the 0.01 and 0.05 level in the Signed Test. Conclusions-The Fishman's hand and wrist skeletal maturation index and Bacetti's cervical vertebrae maturation stages are both useful tools in evaluating growth stages. Fishman's handwrist method is slightly more accurate.
\end{abstract}




\section{Keywords}

Skeletal Maturation, Cervical Vertebrae, Hand-Wrist

\section{Introduction}

An understanding of growth is very important to the practice of clinical orthodontics and dentofacial orthopedics. The growth stage of the patient can have considerable influence on diagnosis, treatment goals, treatment planning, and the eventual outcome of treatment. Clinical decisions regarding use of orthopedic extra-oral traction forces, functional appliances to make orthopedic changes, extraction versus non-extraction treatment, or orthognathic surgery may be dependent upon growth considerations. For example, the use of functional appliances to correct Class II skeletal discrepancies will have its greatest affect if the peak in mandibular growth in a favorable direction occurs during the treatment period [1]. In the light of other studies [2] [3] [4], it is appropriate to take another look at the two most commonly utilized methods of growth evaluation, the hand-wrist radiographic analysis and the cervical vertebrae analysis using the cephalometric radiograph.

The typical growth pattern of a child is characterized by a growth rate that decreases from birth, with a minor mid-growth spurt at approximately 6 to 8 years of age, a pre pubertal minimum, and a pubertal growth spurt [2]. Although growth events occur in this reasonably constant sequence, the chronological ages at which they are reached vary considerably among children. Because of this variation, physiologic parameters have been used in an attempt to identify stages of growth, such as chronological age, sexual maturation characteristics, dental development, attainment of peak growth velocity, height, weight, and skeletal development. Moore et al. [5] in 1990, found a correlation between skeletal maturation and statural height.

The standard method for evaluating skeletal maturity was, for many years, by use of hand-wrist radiographs. In the 1900s, studies were performed tabulating indicators of maturity on sequential radiographs of the growing hand and wrist. In 1928, Hellman [6] published his observations on the ossification of epiphyseal cartilages of the hand. Greulich and Pylep [7] later made a radiographic atlas for the skeletal development of the hand-wrist. A more current system of skeletal maturation indicators (SMIs) was developed in 1979 by Fishman [8] showing that developmental events of an individual's hand relate directly to the progression of maturation during childhood and adolescence. Each progressive stage represents an increasing percentage of total facial skeletal growth completed.

The stages of cervical vertebrae maturation (CVM) are related to growth changes in the mandible, which has also proved to be effective in determining mandibular skeletal maturation in growing subjects according to O'Reilly and Yanniello. [9] Franchi, et al. [10], confirmed the validity of the six CVM stages 
as biologic indicator for both mandibular and somatic skeletal maturity. Franchi's studies showed that the growth interval between stage 3 and stage 4 of CVM coincides with the pubertal peak in mandibular growth and body height.

Several attempts were made at cervical vertebrae analysis beginning in the 1970s [1] [11] with the most frequently used one modified by Bacetti et al. [12] in 2002. The new analysis was comprised of not 6 but 5 stages of CVM. Statistically significant increases in mandibular length, corpus length, and ramus height were associated with specific maturation stages. Since they determined that no statistically significant discrimination can be made between CVMS 1 and CVMS 2 as defined in the former CVM method, they further stated that the presence of a concavity at the lower border of the second cervical vertebra is not a distinctive feature of CVMS 2 when compared to CVMS 1. Thus the two former stages (CVMS 1 and CVMS 2) were merged into one single stage in this method.

\section{Purpose of Study}

The primary purpose of the present study was to analyze associations between the cervical vertebrae maturation stage (CVMS) method proposed by Bacetti, et al. [12], and the hand-wrist skeletal maturation index (SMI), developed by Fishman [8]. The second objective was to determine the reproducibility and consistency of the determinations made on lateral cephalograms and hand-wrist radiographs.

\section{Materials \& Methods}

The cephalometric and hand-wrist radiographs of 92 subjects (44 females and 48 males) from the Postdoctoral Orthodontic clinic at the Columbia University College of Dental Medicine comprised the sample for this study. Subjects were included only if both the lateral cephalometric and hand-wrist radiographs were taken on the same day. None of the patients included had any anatomical malformations of the cervical vertebrae or hand-wrist. The patients had an age range of 8 to 17 years. Poor clarity or contrast of the radiograph excluded the subject from the sample pool.

All the radiographs were taken from the same digital $\mathrm{x}$-ray equipment, Dentsply Gendex, OrthoralixTM 9000 System. Radiographs were evaluated using Gendex Imaging software, VixWin.

The skeletal maturation index (SMI) system developed by Fishman [7] was used to determine skeletal maturation by hand-wrist evaluation for each subject. This system utilizes four ossification stages of bone maturation found at six sites located on the thumb, third finger, fifth finger, and radius. Eleven adolescent skeletal maturation indicators (SMI) are found at these six sites. The sequence of four ossification stages includes epiphyseal widening, ossification of the sesamoid of the thumb, capping of the selected epiphyses over their diaphysis, and fusion of selected epiphyses.

Once the SMI was scored from the hand-wrist radiograph, the lateral cepha- 
logram of the same respective subject, which was taken on the same date, was assessed. The morphology of the cervical vertebrae $\mathrm{C} 2$ through $\mathrm{C} 4$ was evaluated by visual inspection. Cervical Vertebral Maturation Stage (CVMS) was attained using the system described by Bacetti, et al. [11].

Intra-observer error was determined by interpreting the SMI and CVMS of the same records 2 weeks later, and comparing results. The CVMS readings were evaluated against the previously determined SMI readings to see what associations exist.

Statistical analyses were performed using a commercial program, SPSS V. 11.5 for Windows (Manufacturer: SPSS Inc. Chicago, IL 60606). The program generated the mean, standard deviation, minimum, maximum, range, and sample size. Comparisons and correlations were made to obtain a $\mathrm{P}$ value to provide inference for significance at the $\mathrm{P}<0.05$ level.

In an earlier study by Hassel and Farman [13], the cervical vertebrae skeletal maturation was sequentially segregated by SMI grouping. Six categories of cervical vertebrae maturation were defined and related to Fishman's skeletal maturation index (SMI) as shown in Table 1.

In order to explore the associations of the five-stage CVMS to SMI, the following methods were used to determine what associations exist as shown in $\mathrm{Ta}$ ble 2 .

Statistical analyses were performed using a commercial program, SPSS V. 11.5 for Windows (Manufacturer: SPSS Inc. Chicago, IL 60606). The program generated the mean, standard deviation, minimum, maximum, range, and sample

Table 1. CVM stage as related to Fishman's method of hand/wrist analysis.

\begin{tabular}{ccc}
\hline Hand-Wrist SMI & Cervical Vertebral Stage & Percentage of Pubertal Growth Remaining \\
\hline $1-2$ & 1. Initiation & $85 \%-100 \%$ \\
$3-4$ & 2. Acceleration & $65 \%-85 \%$ \\
$5-6$ & 3. Transition & $25 \%-65 \%$ \\
$7-8$ & 4. Deceleration & $10 \%-25 \%$ \\
$9-10$ & 5. Maturation & $5 \%-10 \%$ \\
11 & 6. Completion & $0 \%$ \\
\hline
\end{tabular}

Table 2. Three methods relating H/W analysis to 5 stages of CVMS.

\begin{tabular}{cccccc}
\hline \multicolumn{2}{c}{ Method 1 } & \multicolumn{2}{c}{ Method 2 } & \multicolumn{3}{c}{ Method 3 } \\
\hline Hand-Wrist SMI & CVMS & Hand-Wrist SMI & CVMS & Hand-Wrist SMI & CVMS \\
\hline $1-2$ & I & $1-3$ & I & $1-4$ & I \\
$3-6$ & II & $4-6$ & II & $5-6$ & II \\
$7-8$ & III & $7-8$ & III & $7-8$ & III \\
$9-10$ & IV & $9-10$ & IV & $9-10$ & IV \\
11 & V & 11 & V & 11 & V
\end{tabular}


size. Comparisons and correlations were made to obtain a $\mathrm{P}$ value to provide inference for significance.

This study was approved by the Columbia University Institutional Review Board.

\section{Results}

Descriptive statistics of the variables are found in Table 3.

Intra-observer correlation was high when determining SMI stage. The SMI stage was determined for each of the 92 subjects by evaluation of the hand-wrist radiograph. These same radiographs were reevaluated 2 weeks later by the same operator and the SMI categories were again assigned. Eighty of the 92 SMI determinations were the same in the second evaluation as they were in the first. A high correlation existed between the first and second readings using Spearman's rho coefficient (correlation $=0.993, \mathrm{p}<0.01)($ Table 4). Wilcoxon signed ranks test and the Sign test revealed probability scores of 0.248 and 0.388 , respectively, indicating no significant difference (at $\mathrm{P}<0.05$ or 0.01 ) between the two readings (Table 5).

Table 3. Descriptive statistics of the variables.

\begin{tabular}{|c|c|c|c|c|c|c|c|c|c|c|c|}
\hline & $\mathbf{N}$ & Range & Minimum & Maximum & Mean & & Std. Deviation & Skewness & & Kurtosis & \\
\hline & Statistic & Statistic & Statistic & Statistic & Statistic & $\begin{array}{l}\text { Std. } \\
\text { Error }\end{array}$ & Statistic & Statistic & $\begin{array}{l}\text { Std. } \\
\text { Error }\end{array}$ & Statistic & $\begin{array}{l}\text { Std. } \\
\text { Error }\end{array}$ \\
\hline Sex & 92 & 1 & 1 & 2 & 1.478 & 0.052 & 0.502 & 0.088 & 0.251 & -2.037 & 0.498 \\
\hline Age (rounded) & 92 & 9 & 9 & 18 & 12.750 & 0.208 & 1.998 & 0.404 & 0.251 & -0.438 & 0.498 \\
\hline Hand \& Wrist SMI-1 & 92 & 10 & 1 & 11 & 6.000 & 0.341 & 3.268 & -0.097 & 0.251 & -1.308 & 0.498 \\
\hline Hand \& Wrist SMI-2 & 92 & 10 & 1 & 11 & 5.957 & 0.344 & 3.298 & -0.079 & 0.251 & -1.307 & 0.498 \\
\hline CVM stage-1 & 92 & 4 & 1 & 5 & 2.717 & 0.131 & 1.252 & -0.097 & 0.251 & -1.258 & 0.498 \\
\hline CVM stage-2 & 92 & 4 & 1 & 5 & 2.739 & 0.131 & 1.257 & -0.135 & 0.251 & -1.267 & 0.498 \\
\hline Hand \& Wrist 5 -stage- 1 method 1 & 92 & 4 & 1 & 5 & 2.598 & 0.123 & 1.177 & 0.297 & 0.251 & -0.892 & 0.498 \\
\hline Hand \& Wrist 5 -stage- 2 method 1 & 92 & 4 & 1 & 5 & 2.576 & 0.124 & 1.188 & 0.317 & 0.251 & -0.911 & 0.498 \\
\hline Hand \& Wrist 5-stage- 1 method 2 & 92 & 4 & 1 & 5 & 2.478 & 0.133 & 1.279 & 0.291 & 0.251 & -1.152 & 0.498 \\
\hline Hand \& Wrist 5-stage- 2 method 2 & 92 & 4 & 1 & 5 & 2.467 & 0.133 & 1.279 & 0.316 & 0.251 & -1.140 & 0.498 \\
\hline Hand \& Wrist 5-stage- 1 method 3 & 92 & 4 & 1 & 5 & 2.446 & 0.138 & 1.321 & 0.296 & 0.251 & -1.265 & 0.498 \\
\hline Hand \& Wrist 5-stage-2 method 3 & 92 & 4 & 1 & 5 & 2.413 & 0.138 & 1.319 & 0.340 & 0.251 & -1.229 & 0.498 \\
\hline Hand \& Wrist Average & 92 & 10 & 1 & 11 & 5.978 & 0.342 & 3.278 & -0.088 & 0.251 & -1.305 & 0.498 \\
\hline CVM stage Average & 92 & 4 & 1 & 5 & 2.728 & 0.130 & 1.248 & -0.108 & 0.251 & -1.253 & 0.498 \\
\hline $\begin{array}{l}\text { Hand \& Wrist 5-stage Method } 1 \\
\text { Average }\end{array}$ & 92 & 4 & 1 & 5 & 2.587 & 0.123 & 1.180 & 0.315 & 0.251 & -0.896 & 0.498 \\
\hline $\begin{array}{l}\text { Hand \& Wrist } 5 \text {-stage Method } 2 \\
\text { Average }\end{array}$ & 92 & 4 & 1 & 5 & 2.473 & 0.133 & 1.278 & 0.304 & 0.251 & -1.140 & 0.498 \\
\hline $\begin{array}{c}\text { Hand \& Wrist } 5 \text {-stage Method } 3 \\
\text { Average }\end{array}$ & 92 & 4 & 1 & 5 & 2.429 & 0.137 & 1.317 & 0.319 & 0.251 & -1.243 & 0.498 \\
\hline
\end{tabular}


Table 4. Intra-observer correlation (Spearman's Rho coefficient).

\begin{tabular}{cccccc}
\hline & & N & Correlation & Sig. & Sig. \\
\hline Pair 1 & Hand-Wrist SMI 1 \& 2 & 92 & 0.993 & 0.000 & Yes \\
Pair 2 & CVM stage 1 \& 2 & & 0.981 & 0.000 & Yes \\
\hline
\end{tabular}

Significance level $=0.01$.

Table 5. Intra-observer non-parametric paired sample T-test. (a) Wilkoxin signed ranks test; (b) Sign test.

(a)

\begin{tabular}{ccc}
\hline & H \& W SMI 2-H \& W SMI 1 & CVM stage 2-CVM stage 1 \\
\hline Z & -1.155 & -0.816 \\
Asym. Sig. (2-tailed) & 0.248 & 0.414 \\
Sig. level $<0.05$ & No & No \\
Sig. level $<0.01$ & No & No \\
\hline
\end{tabular}

(b)

\begin{tabular}{ccc}
\hline & H\&W SMI 2-H \& W SMI 1 & CVM stage 2-CVM stage 1 \\
\hline Exact sig. $(2$ tailed) & 0.388 & 0.688 \\
Sig. level $<0.05$ & no & no \\
Sig. level $<0.01$ & no & no \\
\hline
\end{tabular}

High intra-observer correlation existed when assigning CVMS. Lateral cephalometric radiographs from the same 92 patients as the hand-wrist films were evaluated, and CVM stages were assigned. The same radiographs were reevaluated 2 weeks later by the same operator. 88 of the 92 CVM stage determinations coincided with those determined 2 weeks previously. The correlation was also high between the two readings (correlation $=0.981, \mathrm{p}<0.01$ ) using Spearman's rho coefficient (Table 4). Wilcoxon signed ranks test and sign test revealed probability scores of 0.414 and 0.688 , respectively, indicating no significant difference between the two readings (Table 5).

High correlation was found between the hand-wrist and cervical vertebrae readings. The Spearman's rho correlation coefficient was 0.925 and significant at the $\mathrm{P}<0.01$ level (Table 6). The correlation between hand-wrist and age $(0.665$, $\mathrm{p}<0.01)$ was slightly greater than that of the CVMS $(0.611, \mathrm{p}<0.01)$ as shown in Table 6. When the three categorically modified methods (see Materials and Methods) of the Fishman's 11 skeletal maturation stage in hand and wrist were used to compare with CVMS, methods 2 and 3 were both statistically different according to the Wilcoxon signed ranks test and the Sign test at an significance level of $\mathrm{P}<0.05$ (Table 7). However, for method 1, the tests showed probability scores of 0.028 and 0.151 , respectively, showing no significant difference at the $\mathrm{P}$ $<0.01$ level in the Wilcoxon Signed Ranks Test, and no significant difference at the 0.01 and 0.05 level in the Signed Test (Table 7). 
Table 6. CVM, HW-SMI \& age correlations.

\begin{tabular}{cccccc}
\hline & & CVM stage mean & H-W mean & Age (rounded) \\
\hline Spearman rho CVM stage mean & Corr. Coeff. & 1 & 0.925 & 0.611 \\
& Sig. (2-tailed) & & 0.000 & 0.000 \\
& $\mathrm{~N}$ & 92 & 92 & 92 \\
H-W mean & Corr. Coeff. & 0.925 & 1 & 0.665 \\
& Sig. (2 tailed) & 0.000 & & 0.000 \\
& $\mathrm{~N}$ & 92 & 92 & 92 \\
Age (rounded) & Corr. Coeff. & 0.611 & 0.665 & 1 \\
& Sig. (2 tailed) & 0.000 & 0.000 & \\
& $\mathrm{~N}$ & 92 & 92 & 92 \\
\hline
\end{tabular}

Table 7. Comparison of 3 modified H-W SMI with CVMS stage. (a) Wilcoxon signed ranks test; (b) Sign test.

(a)

\begin{tabular}{cccc}
\hline & $\begin{array}{c}\text { Modified H-W } \\
\text { 1-CVMS }\end{array}$ & $\begin{array}{c}\text { Modified H-W } \\
\text { 2-CVMS }\end{array}$ & $\begin{array}{c}\text { Modified H-W } \\
\text { 3-CVMS }\end{array}$ \\
\hline $\mathrm{Z}$ & -2.193 & -3.745 & -4.169 \\
Asymp. Sig. (2 tailed) & 0.028 & 0.000 & 0.000 \\
Sig. level $<0.01$ & No & Yes & Yes \\
Sig. level $<0.05$ & Yes & Yes & Yes \\
\hline
\end{tabular}

(b)

\begin{tabular}{cccc}
\hline & $\begin{array}{c}\text { Modified H-W } \\
\text { 1-CVMS }\end{array}$ & $\begin{array}{c}\text { Modified H-W } \\
\text { 2-CVMS }\end{array}$ & $\begin{array}{c}\text { Modified H-W } \\
\text { 3-CVMS }\end{array}$ \\
\hline Z & -1.437 & -3.592 & -3.833 \\
Asymp. Sig. (2 tailed) & 0.151 & 0.000 & 0.000 \\
Sig. level $<0.01$ & No & Yes & Yes \\
Sig. level $<0.05$ & No & Yes & Yes \\
\hline
\end{tabular}

\section{Discussion}

The high correlation between the cervical vertebral maturation stages and the hand-wrist skeletal maturation indices confirmed previous studies [14] [15] [16] showing that both methods can be utilized to help determine growth potential in an adolescent patient. The main reason for using CVM rather than hand-wrist evaluation has been to reduce radiation exposure since a cephalometric radiograph is readily available because it is required for diagnosis and treatment planning in almost every orthodontic case. However a 2012 study by Patcas et al., [17] has shown that the effective dose of a conventional hand-wrist radiograph added to that of a cephalometric radiograph with a thyroid shield is less than a cephalometric radiograph alone without a thyroid shield. This is because 
the thyroid is very sensitive to radiation exposure and it's shielding significantly reduces the effective dose. Thus, in accordance with the ALARA (as low as reasonably achievable) principle, an additional hand-wrist radiograph seems more justifiable than removing the thyroid shield when taking a cephalometric radiograph.

The observations of Lamparski [11], Hassel and Farman [13], and Bacetti et al. [12], were confirmed during the evaluation of CVMS. The size, shape, and inferior curvature were seen to differ at each level of maturation. The vertebral bodies changed from wedge-shaped, to horizontal rectangular, to square, to vertical rectangular as skeletal maturity progressed. The inferior borders changed from flat to concave, and were seen to appear sequentially from $\mathrm{C} 2$ to $\mathrm{C} 3$ to $\mathrm{C} 4$ during maturational progression, and became more distinct. However, difficulty determining classification occurred at times. The difficulty occurred when differentiating borderline cases. It must be kept in mind that skeletal maturation is a continuous process with each stage of maturation blending into the next.

The evaluation and interpretation of the CVMS of the same case was consistent, as shown by the high intra-observer correlation. The evaluation of intraoperator error in SMI determination also suggested that interpretation from one time to the next varied slightly but not significantly. The instances of intra-observer disagreement, in this study, fell within one CVMS category of the previous interpretation. Clinically, these differences should not be of great importance. However, in this study, only one evaluator's subjective interpretation of Fishman and Bacetti's staging methods was used. It may be useful to have multiple evaluators classify the SMI and CVMS of these subjects to determine if an inter-observer discrepancy would exist.

The modification of Hassel and Farman's 6-stage CVS [16] to Bacetti's, et al., 5-stage CVMS poses a few problems when trying to correlate CVMS to SMI. Bacetti combined the original CVS 1 and 2 into one stage, CVMS I. Therefore, according to Farman and Hassel, this one stage should correlate with SMI 1 through 4. However, Bacetti's CVMS II definition states that the peak in mandibular growth will occur within one year after this stage. According to this, CVMS II should include the SMI 4, sesamoid ossification, since this stage has proven to occur on an average of one year before peak in mandibular growth.

In this study, three categorically modified methods were used to compare CVMS and SMI in an attempt to see which method of SMI to CVMS would correlate the best. In method \#1, CVMS I was considered similar to SMI 1 and 2 and CVMS II included SMI 3 through 6. In Method \#2, CVMS I corresponded with SMI 1 to 3, and CVMS II corresponded with SMI 4 to 6. In Method \#3, CVMS I corresponded with SMI 1 through 4, and CVMS II with SMI 5 and 6. Some limitations of this study include the fact that, to achieve more accurate results, a more specific correlation test would have to be performed consisting of the categorical combinations of SMI's in methods 1, 2, and 3, versus CVMS I and II only, excluding CVMS III, IV, and V. We considered this to be a limitation of 
the study, however, in the long run, we concluded that doing this would have added too much irrelevant data to the study. Also, the male and female subjects could have been measured separately to determine whether there was sexual dimorphism. We also think that it would be useful to repeat Hassel and Farman's [13] study and using Bacetti's CVMS method to observe the relations between CVMS I and SMI stages 1 through 6. In summary, the discrepancy between the modified methods and CVMS in the significant levels demonstrates a wider range of questionable compatibility to merge the two methodologies as one. In a 2013 study Beit [18] et al. found that CVM offers no advantage over chronologic age in assessing skeletal age or in predicting the pubertal growth spurt.

The study by Turchetta et al. [2] demonstrated that a maturation-based analysis was better than a chronologically based method such as the Johnston grid [19] or the Ricketts [20] analysis for both long term and short term growth prediction.

\section{Conclusions}

1) The Fishman hand and wrist skeletal maturation index and Bacetti's cervical vertebrae maturation stages are both effective for evaluating craniofacial growth and development to facilitate orthodontic and orthopedic diagnosis and treatment planning.

2) The correlation between hand-wrist SMI and age $(0.665, \mathrm{p}<0.01)$ was slightly greater than that of the CVMS $(0.611, \mathrm{p}<0.01)$.

3) In this study, the hand-wrist analysis was slightly more accurate than CVMS in evaluation of growth and development.

\section{References}

[1] Haag, U. and Taranger, J. (1982) Maturation Indicators and the Pubertal Growth Spurt. American Journal of Orthodontics, 82, 299-309. https://doi.org/10.1016/0002-9416(82)90464-X

[2] Turchetta, B.J., Fishman, L.S. and Subtelny, D.J. (2007) Facial Growth Prediction: A Comparison of Methodologies. American Journal of Orthodontics. \& Dentofacial Orthopedics, 132, 439-449. https://doi.org/10.1016/j.ajodo.2005.10.026

[3] Demirijian, A., Buschang, R. and Petterson, K. (1985) Interrelationships among Measures of Somatic, Skeletal, Dental, and Sexual Maturity. American Journal of Orthodontics, 88, 433-438. https://doi.org/10.1016/0002-9416(85)90070-3

[4] Mellon, Z.J., Behrents, R.G. and Johnston, L.E. (2013) The Pattern of Facial Skeletal Growth and Its Relationship to Various Common Indexes of Maturation. American Journal of Orthodontics. \& Dentofacial Orthopedics, 143, 845-854. https://doi.org/10.1016/j.ajodo.2013.01.019

[5] Moore, R.N., Moyer, B.A. and DuBois, L.M. (1990) Skeletal Maturation and Craniofacial Growth. American Journal of Orthodontics and Dentofacial Orthopedics, 98, 33-40. https://doi.org/10.1016/0889-5406(90)70029-C

[6] Hellman, M. (1928) Ossification of Epiphysial Cartilages in the Hand. American Journal of Physical Anthropology, 11, 223-257.

[7] Greulich, W.W. and Pyle, S.I. (1959) Radiographic Atlas of Skeletal Development of 
the Hand-Wrist. Stanford University Press, Stanford.

[8] Fishman, L.S. (1979) Chronological versus Skeletal Age, an Evaluation of Craniofacial Growth. Angle Orthodontist, 49, 181-187.

[9] O’Reilly, M. and Yanniello, G.J. (1988) Mandibular Growth Changes and Maturation of Cervical Vertebrae: A Longitudinal Cephalometric Study. Angle Orthodontist, 58, 179-184.

[10] Franchi, L., Baccetti, T. and McNamara Jr., J.A. (2000) Mandibular Growth as Related to Cervical Vertebral Maturation and Body Height. American Journal of Orthodontics. \& Dentofacial Orthopedics, 118, 335-340.

https://doi.org/10.1067/mod.2000.107009

[11] Lamparski, D.G. (1972) Skeletal Age Assessment Utilizing Cervical Vertebrae. Master of Science Thesis, University of Pittsburgh, Pittsburgh.

[12] Baccetti, I., Franchi, L. and McNamara Jr., J.A. (2002) An Improved Version of the Cervical Vertebral Maturation (CVM) Method for the Assessment of Mandibular Growth. Angle Orthodontist, 72, 316-323.

[13] Hassel, B. and Farman, A.G. (1995) Skeletal Maturation Evaluation Using Cervical Vertebrae. American Journal of Orthodontics. \& Dentofacial Orthopedics, 107, 58-66. https://doi.org/10.1016/S0889-5406(95)70157-5

[14] Garcia-Fernandez, P., Torre, H., Flors, L. and Rea, J. (1998) The Cervical Vertebrae as Maturational Indicators. Journal of Clinical Orthodontics, 32, 221-225.

[15] Grave, K.C. and Brown, T. (1976) Skeletal Ossification and the Adolescent Growth Spurt. American Journal of Orthodontics, 69, 611-619. https://doi.org/10.1016/0002-9416(76)90143-3

[16] Fishman, L.S. (1982) Radiographic Evaluation of Skeletal Maturation: A Clinically Orientated Method Based on Hand Wrist Films. Angle Orthodontics, 52, 88-112.

[17] Patcas, R., Signorelli, L., Peltomaki, T. and Schatzle, M. (2013) A Comparison of Radiation Dose of Two Strategies for Skeletal Age Estimation. European Journal of Orthodontics, 35, 604-660. https://doi.org/10.1093/ejo/cjs043

[18] Beit, P., Peltomaki, T., Schatzle, M., Signorelli, L. and Patcas, R. (2013) Evaluating the Agreement of Skeletal Age Assessment Based on Hand-Wrist and Cervical Vertebrae Radiography. American Journal of Orthodontics and Dentofacial Orthopedics, 144, 838-847. https://doi.org/10.1016/j.ajodo.2013.07.015

[19] Johnston, L.E. (1975) A Simplified Approach to Prediction. American Journal of Orthodontics and Dentofacial Orthopedics, 67, 253-257. https://doi.org/10.1016/0002-9416(75)90048-2

[20] Rickettes, R.M. (1957) Treatment on the Basis of the Facial Pattern and an Estimate of Growth. Angle Orthodontist, 27, 14-37. 